\title{
Acute Kidney Injury Reduces Survival in Very Low Birth Weight Infants
}

\author{
RAJESH KORALKAR, NAMASIVAYAM AMBALAVANAN, EMILY B. LEVITAN, GERALD MCGWIN, STUART GOLDSTEIN, \\ AND DAVID ASKENAZI
}

\begin{abstract}
Department of Pediatrics [R.K., N.A., D.A.], Department of Epidemiology [E.L., G.M.], University of Alabama at Birmingham, Birmingham, Alabama 35205; Department of Pediatrics [S.G.], Cincinnati Children's Hospital and Medical Center, Cincinnati, Ohio 45229
\end{abstract}

\begin{abstract}
Acute kidney injury (AKI) independently predicts mortality in children and adults. Our understanding of the epidemiology of AKI in very LBW (VLBW) infants is limited to retrospective studies. After adjustment for demographics, comorbidities, and interventions, infants with AKI have decreased survival compared with those without AKI. The study was conducted in regional quaternary care NICU of the University of Alabama at Birmingham. VLBW infants were followed prospectively and were classified into a serum creatinine ( $\mathrm{SCr}$ )-based classification for AKI. Forty-one of 229 (18\%) VLBW infants developed AKI. Those with AKI were more likely to have umbilical artery catheters, assisted ventilation, blood pressure medications, and lower 1and 5-min Apgar scores. Of the infants with AKI, 17 of 41 (42\%) died compared with 9 of $188(5 \%)$ of those without AKI $(p<0.001)$. AKI was associated with mortality with a crude hazard ratio (HR) of 9.3 (95\% CI, 4.1-21.0). After adjusting for potential confounders, those with AKI had higher chance of death as the adjusted HR was $2.4(95 \% \mathrm{CI}$ 0.95-6.04). AKI is associated with mortality in VLBW infants. Efforts to prevent and ameliorate the impact of AKI may improve the outcomes in this vulnerable population. (Pediatr Res 69: 354-358, 2011)
\end{abstract}

A dvancements in perinatal medicine have improved survival of critically ill neonates; however, many infants do not survive and others sustain morbidity due to permanent damage to vital organs. In critically ill children and adults, serum creatinine ( $\mathrm{SCr}$ ) changes as low as $0.3 \mathrm{mg} / \mathrm{dL}(26.5 \mu \mathrm{M})$ have been shown to be associated with mortality after correcting for demographics, comorbidities, and severity of illness (1-4). Acute kidney injury (AKI), previously referred to as acute renal failure, may impact survival to a greater degree in premature infants in context of ongoing kidney development (5).

Premature infants are at significant risk for AKI because of high rates of infection and exposure to nephrotoxic medications (6); however, the exact incidence of AKI in this population is unknown. The incidence of neonatal AKI, estimated at $8-24 \%$ of the general neonatal population, is probably an under-estimate as

Received July 23, 2010; accepted October 30, 2010

Correspondence: David Askenazi, MD, Division of Nephrology, Department of Pediatrics, University of Alabama at Birmingham, 1600 7th Avenue South, ACC 516, Birmingham, AL 35233; e-mail: daskenazi@peds.uab.edu

Supported by the clinical research Young Investigator's Grant of National Kidney foundation, Kaul Pediatric Research Institute Research grant, and O'Brien Kidney Research Center Grant [D.A.].

This study is registered in the national registry of clinical trials (no. NCT00573079) at ClinicalTrials.gov. they only describe those with nonoliguric renal failure and use either high levels of SCr or dialysis provision to define AKI $(7,8)$.

Similarly, outcome data after AKI in premature infants are scant. A recent matched case-control study of premature infants showed that even after adjusting for potential confounders, every $1 \mathrm{mg} / \mathrm{dL}(88.4 \mu \mathrm{M})$ increase in $\mathrm{SCr}$ was associated with 2-fold higher odds of death (9). Inferences about outcomes from this and similar analyses are limited by the retrospective nature of the studies.

We performed a prospective study of very LBW (VLBW) infants (birth weight $\leq 1500 \mathrm{~g}$ ) to improve our understanding of the incidence, outcomes, and independent association between AKI and mortality. AKI was classified using a SCr-based categorical definition similar to Acute Kidney Injury Network (AKIN) (10) (Table 1). The AKIN definition and the pediatric risk, injury, failure, loss and end-stage (pRIFLE) definition have been evaluated in several pediatric populations including those who required intensive care $(1,2,11)$, received aminoglycosides (12), and required care in a pediatric burn unit (13). Using changes in SCr, these two definitions have been shown to have very close correlation in pediatrics (12). We hypothesized that after adjustment for demographics, comorbidities, and interventions, infants with AKI would have decreased survival compared with those without AKI.

\section{PATIENTS AND METHODS}

The study was conducted in regional quaternary care NICUs of the University of Alabama at Birmingham (UAB) and the Children's Hospital of Alabama. Parental consent was obtained, and the study was approved by the Institutional Review Board at UAB. VLBW infants (birth weight between 500 and $1500 \mathrm{~g}$ ) were enrolled over 18 consecutive months between February 2008 and July 2009. Infants were excluded if they did not survive to $48 \mathrm{~h}$ of life or if they had any significant known congenital abnormality of the kidney.

Study design. We prospectively followed the enrolled infants from the time of birth until 36 wk postmenstrual age (PMA) or hospital discharge, whichever occurred first. SCr was analyzed from remnant serum samples by mass spectrometer and from the patient's medical records from laboratory (measured by Jaffee reaction) performed as part of routine hospital care. A SCr-based classification definition similar to the AKIN (14) definition for AKI was used to classify patients according to severity in three stages (Table 1). Modification of the AKIN classification to this population included a) not using urine output criteria as premature infants often have nonoliguric renal failure due to immature tubular development, and b) an upper cutoff of SCr $2.5 \mathrm{mg} / \mathrm{dL}(221.0 \mu \mathrm{M})$ was chosen to reflect comparable severe kidney dysfunction listed in class 3 of AKIN for

Abbreviations: AKI, acute kidney injury; AKIN, Acute Kidney Injury Network; HR, hazard ratio; PMA, postmenstrual age; VLBW, very LBW; SCr, serum creatinine 
Table 1. Categorical definition of neonatal AKI

\begin{tabular}{cc}
\hline Stage & \multicolumn{1}{c}{ Serum creatinine } \\
\hline AKI 1 & $\uparrow \mathrm{SCr} \geq 0.3 \mathrm{mg} / \mathrm{dL}(26.5 \mu \mathrm{mol} / \mathrm{L})$ from previous value \\
& within $48 \mathrm{~h}$ \\
& $\uparrow \mathrm{SCr} \geq 150-200 \%$ from previous value \\
AKI 2 & $\uparrow \mathrm{SCr} \geq 200-300 \%$ from previous value \\
AKI 3 & $\uparrow \mathrm{SCr} \geq 300 \%$ from previous value or $\mathrm{SCr} \geq 2.5 \mathrm{mg} / \mathrm{dL}$ \\
& $(221.0 \mu \mathrm{mol} / \mathrm{L})$ or need for dialysis \\
\hline
\end{tabular}

Table 2. Demographic variables, comorbidities, and interventions in enrolled infants

\begin{tabular}{|c|c|c|c|}
\hline & $\begin{array}{c}\text { No AKI } \\
(n=188)\end{array}$ & $\begin{array}{c}\text { AKI } \\
(n=41)\end{array}$ & $p$ \\
\hline \multicolumn{4}{|l|}{ Infant characteristics } \\
\hline GA $(w k) *$ & $28 \pm 3$ & $25 \pm 2$ & $<0.001$ \\
\hline Birth weight $(\mathrm{g}) *$ & $1039 \pm 279$ & $702 \pm 205$ & $<0.001$ \\
\hline Male sex & $91(48 \%)$ & $17(42 \%)$ & 0.42 \\
\hline Race & & & 0.8 \\
\hline Black & $98(52 \%)$ & $19(46 \%)$ & \\
\hline White & $78(41.5 \%)$ & $19(46 \%)$ & \\
\hline Hispanic & $12(6.4 \%)$ & $3(7 \%)$ & \\
\hline 1-min Apgar $\ddagger$ & $4.6 \pm 0.2$ & $3 \pm 0.3$ & 0.02 \\
\hline 5-min Apgar & $7 \pm 0.1$ & $6 \pm 0.2$ & $<0.001$ \\
\hline Small-for-GA & $61(33 \%)$ & $15(37 \%)$ & 0.61 \\
\hline \multicolumn{4}{|l|}{ Maternal characteristics } \\
\hline Age $(y)^{*}$ & $25.6 \pm 6$ & $25.8 \pm 6$ & 0.19 \\
\hline Prenatal care & $116(61.7 \%)$ & $28(68 \%)$ & 0.68 \\
\hline Diabetes & $14(8 \%)$ & $3(7 \%)$ & 0.97 \\
\hline Hypertension & $81(43 \%)$ & $11(27 \%)$ & 0.05 \\
\hline Antenatal steroids & $159(85 \%)$ & $32(78 \%)$ & 0.3 \\
\hline Antenatal indomethacin & $2(1 \%)$ & $2(5 \%)$ & 0.1 \\
\hline Smoking & $17(9 \%)$ & $1(2 \%)$ & 0.2 \\
\hline Preeclampsia & $65(35 \%)$ & $7(17 \%)$ & 0.02 \\
\hline Multiple birth & $44(24 \%)$ & $9(22 \%)$ & 0.4 \\
\hline History of drug abuse & $5(3 \%)$ & $3(7 \%)$ & 0.15 \\
\hline Chorioamnionitis & $13(7 \%)$ & $1(2 \%)$ & 0.27 \\
\hline Received antibiotics & $99(53 \%)$ & $23(56 \%)$ & 0.8 \\
\hline Cord $\mathrm{pH}^{*}$ & $7.29 \pm 0.08$ & $7.28 \pm 0.10$ & 0.28 \\
\hline \multicolumn{4}{|l|}{ Interventions } \\
\hline High frequency ventilation & $7(4 \%)$ & $12(29.3 \%)$ & $<0.001$ \\
\hline Conventional ventilation & $94(51 \%)$ & $36(88 \%)$ & $<0.001$ \\
\hline Inotrope use & $26(14 \%)$ & $23(56 \%)$ & $<0.001$ \\
\hline Umbilical arterial catheter & $70(37 \%)$ & $27(66 \%)$ & 0.03 \\
\hline
\end{tabular}

adults $4.0 \mathrm{mg} / \mathrm{dL}(353.6 \mu \mathrm{M})$. Individual infants' baseline creatinine value was taken as first creatinine done on infant. Subsequent values were compared with the lowest previous $\mathrm{SCr}$ measured.

Infant demographic data collected included GA, birth weight, race, sex, and Apgar scores at 1 and $5 \mathrm{~min}$. Maternal demographic data collected included age, diabetes, pregnancy induced hypertension, use of antenatal steroids, presence of multiple births, and known drug use. Comorbid conditions included hyaline membrane disease (HMD), patent ductus arteriosus (PDA), any grade intraventricular hemorrhage (IVH), cardiac disease, cholestasis, presence of necrotizing enterocolitis (NEC), intestinal perforation, and documented bacteria/fungal infections. Interventions documented include use of umbilical arterial catheters, diuretics, inotropes, type of ventilation, and indomethacin. The primary outcome was survival to discharge or 36 wk PMA, whichever occurred first.

Statistical methods. Descriptive statistics were performed to determine differences between VLBW infants with and without AKI (Table 2). Shapiro-Wilk test and normal probability plot were used to test for normality of data. Normally distributed continuous variables were compared using student $t$ test, and nonnormally distributed variables were analyzed using Mann-Whitney $U$ test. Categorical variables were analyzed using $\chi^{2}$ analysis if two variables were present and using Mantel-Haenszel $\chi^{2}$ if three or more categorical variables were present. For all descriptive statistics, $p$ value $<0.05$ was considered statistically significant.

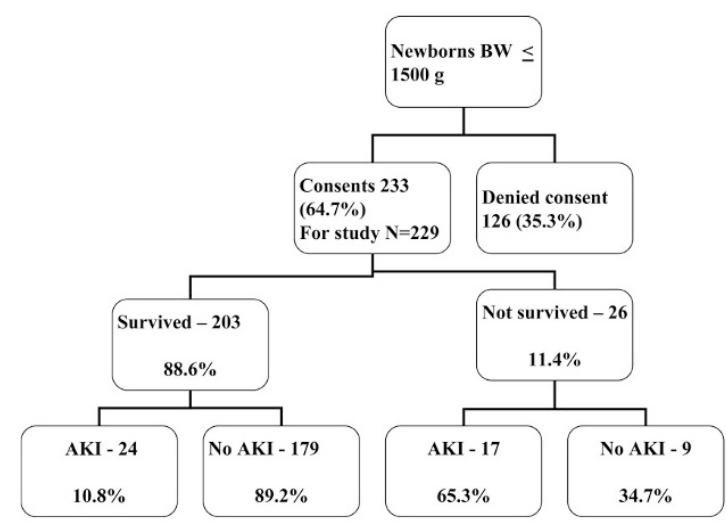

Figure 1. Flow chart depicting enrollment and survival in infants with birth weight $\leq 1500 \mathrm{~g}$ during the 18 -mo prospective evaluation.

The association between AKI and survival were initially analyzed using crude univariate analysis. Then, Cox proportional hazard analysis modeling was performed to control for potentially confounding variables. We adjusted for infant and maternal characteristics and interventions performed on the infant which were suspected to be related to both AKI and infant mortality. For parsimony, our final model only included variables that remained significant at $p<0.10$ in the multivariable-adjusted model. SAS 9.2 (SAS Institute Inc., Cary, NC) was used for the all statistical analysis.

\section{RESULTS}

Of the 360 parental caregivers approached for enrollment, $233(64.7 \%)$ agreed to allow their infant to participate in the study. Of the 233 infants enrolled, four were excluded because they were discharged to another facility before 36 wk PMA; thus, 229 infants were available for analysis (Fig. 1). The earliest SCr measurement was taken was d 1 in 50\% of infants, and in study population, the $\mathrm{SCr}$ was measured on median $\mathrm{d} 4$ (range, 1-48).

AKI was found in 41 of $229(18 \%)$ with 10,10 , and 21 fulfilling criteria for stage 1,2 , and 3 , respectively. No patients received dialysis. Table 2 describes differences in the patient characteristics and comorbidities between those with and those without AKI. Infants with AKI had lower birth weight (mean $702 \mathrm{~g}$ versus $1039 \mathrm{~g}$ ) and lower GA (mean $25 \mathrm{wk}$ versus $28 \mathrm{wk}$ ). Infants with AKI were more likely to have lower 1- and 5-min Apgar scores and require umbilical arterial catheters, assisted ventilation, and inotropes support. Preeclampsia and maternal hypertension had lower AKI rates. Most infants with AKI were $\leq 750 \mathrm{~g}$ [29 of 41 (70\%); Table 3] and $\leq 26$-wk gestation [30 of 41 (73\%); Table 4]. Most episodes of AKI occurred in first week of life [33 of $41(80.5 \%)$ ].

Of those with AKI, 17 of 41 (42\%) died, compared with only 9 of $188(5 \%)$ of those without AKI (Fig. 2). AKI was strongly associated with mortality with an unadjusted Hazard ratio (HR) of 9.3 (95\% CI, 4.1-21.0; $p<0.001)$. Other significant predictors of mortality included GA, birth weight, preeclampsia, exposure to respiratory support, and 1-min and 5-min Apgar scores (Table 5). When these factors were entered into multivariate analysis to control for potential confounding variables, AKI did not remain a predictor for mortality adjusted HR 2.46 (95\% CI, 0.95-6.04; $p<0.07$; Table 6). 
Table 3. Incidence of AKI and mortality by birth weight category

\begin{tabular}{|c|c|c|c|c|c|}
\hline Birth weight & $\begin{array}{l}\leq 750 \mathrm{~g} \\
(n=64)\end{array}$ & $\begin{array}{c}750-1000 \mathrm{~g} \\
(n=62)\end{array}$ & $\begin{array}{c}1000-1250 \mathrm{~g} \\
(n=51)\end{array}$ & $\begin{array}{c}1250-1500 \mathrm{~g} \\
(n=52)\end{array}$ & $p$ \\
\hline No AKI & $35(18.6 \%)$ & $54(28.7 \%)$ & $48(25.5 \%)$ & $51(27.1 \%)$ & $<0.001$ \\
\hline AKI 1 & $6(60 \%)$ & $2(20 \%)$ & $2(20 \%)$ & 0 & \\
\hline AKI 2 & $8(80 \%)$ & $2(20 \%)$ & 0 & 0 & \\
\hline AKI 3 & $15(71.4 \%)$ & $4(19 \%)$ & $1(4.8 \%)$ & $1(4.8 \%)$ & \\
\hline Mortality & $21(80 \%)$ & $3(12 \%)$ & $1(4 \%)$ & $1(4 \%)$ & $<0.001$ \\
\hline
\end{tabular}

Table 4. Incidence of AKI and mortality by GA category

\begin{tabular}{lccccc}
\hline \multicolumn{1}{c}{ GA } & $\begin{array}{c}\leq 26 \mathrm{wk} \\
(n=69)\end{array}$ & $\begin{array}{c}26-28 \mathrm{wk} \\
(n=43)\end{array}$ & $\begin{array}{c}28-30 \mathrm{wk} \\
(n=63)\end{array}$ & $\begin{array}{c}>30 \mathrm{wk} \\
(n=54)\end{array}$ \\
\hline No AKI & $39(20.7 \%)$ & $38(20.2 \%)$ & $59(31.4 \%)$ & $52(27.7 \%)$ & $1(10 \%)$ \\
AKI 1 & $7(70 \%)$ & $1(10 \%)$ & $1(10 \%)$ & 0 & $<0.001$ \\
AKI 2 & $9(90 \%)$ & $1(10 \%)$ & 0 & $1(4.8 \%)$ & 0 \\
AKI 3 & $14(56.7 \%)$ & $3(14.3 \%)$ & $3(14.3 \%)$ & $<0.001$ \\
Mortality & $22(84 \%)$ & $1(4 \%)$ & $3(12 \%)$ & \\
\hline
\end{tabular}

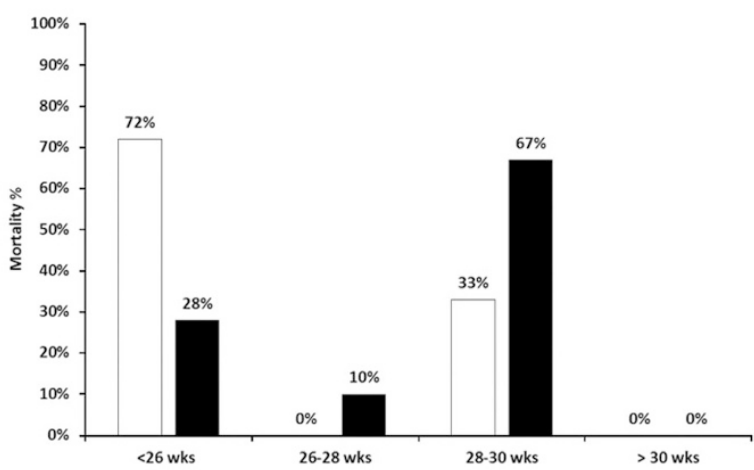

Figure 2. Incidence of mortality by GA category given AKI. AKI: $\square$; No AKI:

Table 5. Predictors of mortality among 229 very LBW infants

\begin{tabular}{lcc}
\hline & Crude Hazard ratio (95\% CI)* & $p$ \\
\hline Race & $0.93(0.49-1.78)$ & 0.83 \\
Gender & $0.45(0.20-1.01)$ & 0.45 \\
GA & $0.50(0.39-0.64)$ & $<0.001$ \\
Birth weight & $0.53(0.42-0.67)$ & $<0.001$ \\
Prenatal care & $1.40(0.58-3.40)$ & 0.27 \\
Preeclampsia & $0.18(0.04-0.75)$ & 0.02 \\
Hypertension & $0.44(0.17-1.10)$ & 0.08 \\
Maternal age & $0.98(0.92-1.05)$ & 0.25 \\
1-Min Apgar & $0.77(0.65-0.92)$ & 0.003 \\
5-Min Apgar & $0.70(0.60-0.83)$ & $<0.001$ \\
High frequency ventilation & $9.12(4.20-20.00)$ & $<0.001$ \\
Conventional ventilation & $17.1(2.30-126.50)$ & $<0.005$ \\
Umbilical arterial catheter & $1.9(0.80-4.20)$ & 0.11 \\
Multiple birth & $1.43(0.60-3.30)$ & 0.40 \\
Small-for-GA & $1.21(0.54-2.72)$ & 0.64 \\
\hline
\end{tabular}

* Cox Proportional Hazard ratios for race compared with white, Gender female compared with male, GA per day increase, Birth weight per $100 \mathrm{~g}$ increase, Apgar scores per point increase and for categorical variables present compared with absent.

\section{DISCUSSION}

To our knowledge, this is the first prospective epidemiologic study on AKI in VLBW infants that attempts to control for potential confounders. This analysis also represents the first attempt to classify this population according to severity of
AKI similar to definitions used in pediatric and adult studies (14). AKI was strongly associated with mortality, even after adjustment for patient characteristics and potential confounders, indicating that it is an independent risk factor and not just a marker for illness severity.

Our finding corroborates conclusions from similar studies in neonates which show the negative association between AKI and survival. Mathur et al. (15) prospectively studied term neonates with sepsis and found a $26 \%$ incidence of AKI and also identified higher mortality in those who had AKI compared with those without AKI (no adjustment for potential confounders was performed in that analysis). A recent retrospective matched case-control study of premature infants $(\leq 1000 \mathrm{~g})$ showed that an increase in $\mathrm{SCr}$ of $1.0 \mathrm{mg} / \mathrm{dL}(88.4 \mu \mathrm{M})$ doubled the odds of death even after adjustment for important confounders.

Compared with other pediatric and adult studies, our analysis showed a greater proportion of stage 3 AKI in those who developed AKI. This is possibly because premature infants start with very low glomerular filtration rate (GFR) and thus mild exposure could cause high degree of injury. Alternatively, this could be due to our study design. Our incidence represents a "best-case scenario" as we did not have access to daily SCr levels on these infants and many did not have any SCr levels available. We only had access to laboratories from those infants in which clinician had ordered the test or if infants warranted blood gas analysis (and subsequent remnant blood was available). Thus, our population could have been selected for the most severely ill patients.

Our conclusions are similar to other critically ill pediatric $(1,2,13)$ and adult $(3,16,17)$ populations, which show an independent association between AKI and mortality. A large meta-analysis of $>20,000$ adult patients by Ricci et al. (18) showed adjusted relative risks of death of 2.4, 4.2, and 6.4 for mild, moderate, and severe AKI, respectively $(p<0.0001$ for all). Our study found a similar increase in the likelihood of death with AKI, but we did not find increased mortality with increasing AKI stage. This could be explained by a) the 
Table 6. Difference in survival between infants with AKI and without AKI

\begin{tabular}{|c|c|c|c|c|}
\hline & $\begin{array}{c}\text { Survival } \\
(N=203)\end{array}$ & $\begin{array}{c}\text { Death } \\
(N=26)\end{array}$ & $\begin{array}{c}\text { Crude HR } \\
(95 \% \text { CI })\end{array}$ & $\begin{array}{l}\text { Adjusted HR } \\
(95 \% \mathrm{CI})^{*}\end{array}$ \\
\hline \multicolumn{5}{|l|}{ Any AKI } \\
\hline No AKI & $179(95 \%)$ & $9(5 \%)$ & Ref & Ref \\
\hline Any AKI & $24(58 \%)$ & $17(42 \%)$ & $9.3(4.1-21.0)$ & $2.46(0.95-6.04)$ \\
\hline \multicolumn{5}{|l|}{ AKI category } \\
\hline AKI 1 & $7(70 \%)$ & $3(30 \%)$ & $6.8(1.8-25.0)$ & $2.87(0.72-11.44)$ \\
\hline AKI 2 & $7(70 \%)$ & $3(30 \%)$ & $6.1(1.6-22.2)$ & $1.37(0.35-5.41)$ \\
\hline AKI 3 & $10(48 \%)$ & $11(52 \%)$ & $12.4(5.1-30.1)$ & $3.13(1.10-8.86)$ \\
\hline
\end{tabular}

* Controlled for GA, birth weight, high frequency ventilation, 5-min Apgar.

association of baseline SCr levels in neonates initially reflecting maternal $\mathrm{SCr}, \mathrm{b})$ relatively high death rate in those with AKI classification 1 compared with other populations that could be related to early mortality (before AKI class 2 could be documented), or c) lack of documentation of transition from stage 1 to 2 due to lack of daily SCr in most of infants.

The incidence of AKI was found to be $18 \%$ of the cohort, with highest rates in the most premature infants. Because we did not measure SCr on every baby, every day it is possible that these rates may be even higher. Comparatively, retrospective studies have found an $8-24 \%$ incidence rate in the heterogeneous neonatal population $(7,8)$.

Although this study was not designed to explore the variables that predispose or protect against AKI, infants born to mothers with preeclampsia and/or maternal hypertension were protected from AKI. If this finding is consistent, a more robust risk factor analysis of these data, and in other neonatal databases, elucidating the etiology of these findings may enhance our understanding of the pathophysiology of protective AKI mechanisms.

The prospective design of our study limits potential bias, but some important limitations need to be acknowledged. First, we had to rely on measurements of SCr performed as part of clinical care or from remnant serum, when available. Therefore, the true incidence may be even higher than what we have reported. Second, our findings are limited by our definition of $\mathrm{AKI}$, which relies on $\mathrm{SCr}$ changes to diagnose AKI. Not only is $\mathrm{SCr}$ a poor marker of injury, but also the normal decrease in $\mathrm{SCr}$, which normally occurs in the first weeks of life, makes it even more difficult to interpret in neonates (reviewed in Ref. 19). This and other problems with using changes in SCr as gold standard to diagnose AKI limit our ability to reliably provide incidence and outcome data. As novel urine injury biomarkers are validated in this population, reevaluation of the impact of acute increases in these biomarkers (20-23) may improve our understanding of the incidence and outcome in this population.

Although we attempted to control for the most predictive variables of mortality seen in our population, some measured and/or unmeasured variables may have been missed in our analysis. The adjusted HR is nonetheless robust, which likely means the independent association between AKI and mortality is real. Future studies on the impact of AKI on survival may be improved using a matched prospective cohort study (matching for GA, birth weight, and severity of illness) and collaboration between multiple centers.

In conclusion, AKI is common in VLBW and carries a high mortality risk after controlling for demographics, comorbidities, and interventions. Most infants who developed AKI were extremely premature and developed AKI within the first week of life. Efforts to prevent and ameliorate the impact of AKI are likely to improve the outcomes in this vulnerable population. Large multicenter studies are needed to corroborate these findings.

Acknowledgments. We thank Amy Logue, RN, BSN, for her assistance in data collection.

\section{REFERENCES}

1. Akcan-Arikan A, Zappitelli M, Loftis LL, Washburn KK, Jefferson LS, Goldstein SL 2007 Modified RIFLE criteria in critically ill children with acute kidney injury. Kidney Int 71:1028-1035

2. Plötz FB, Bouma AB, van Wijk JA, Kneyber MC, Bokenkamp A 2008 Pediatric acute kidney injury in the ICU: an independent evaluation of pRIFLE criteria. Intensive Care Med 34:1713-1717

3. Hoste EA, Kellum JA 2006 Acute kidney injury: epidemiology and diagnostic criteria. Curr Opin Crit Care 12:531-537

4. Chertow GM, Burdick E, Honour M, Bonventre JV, Bates DW 2005 Acute kidney injury, mortality, length of stay, and costs in hospitalized patients. J Am Soc Nephrol $16: 3365-3370$

5. Rodríguez MM, Gomez AH, Abitbol CL, Chandar JJ, Duara S, Zilleruelo GE 2004 Histomorphometric analysis of postnatal glomerulogenesis in extremely preterm infants. Pediatr Dev Pathol 7:17-25

6. Askenazi DJ, Feig DI, Graham NM, Hui-Stickle S, Goldstein SL 2006 3-5 year longitudinal follow-up of pediatric patients after acute renal failure. Kidney Int 69:184-189

7. Stapleton FB, Jones DP, Green RS 1987 Acute-renal-failure in neonates-incidence, etiology and outcome. Pediatr Nephrol 1:314-320

8. Agras PI, Tarcan A, Baskin E, Cengiz N, Gurakan B, Saatci U 2004 Acute renal failure in the neonatal period. Ren Fail 26:305-309

9. Askenazi DJ, Griffin R, McGwin G, Carlo W, Ambalavanan N 2009 Acute kidney injury is independently associated with mortality in very low birthweight infants: a matched case-control analysis. Pediatr Nephrol 24:991-997

10. Mehta RL, Kellum JA, Shah SV, Molitoris BA, Ronco C, Warnock DG, Levin 2007 A Acute Kidney Injury Network (AKIN): report of an initiative to improve outcomes in acute kidney injury. Crit Care 11:R31

11. Ozcakar ZB, Yalcinkaya F, Altas B, Ergun H, Kendirli T, Ates C, Elhan AH, Ekim M 2009 Application of the new classification criteria of the Acute Kidney Injury Network: a pilot study in a pediatric population. Pediatr Nephrol 24:1379-1384

12. Zappitelli M, Parikh CR, Akcan-Arikan A, Washburn KK, Moffett BS, Goldstein SL 2008 Ascertainment and epidemiology of acute kidney injury varies with definition interpretation. Clin J Am Soc Nephrol 3:948-954

13. Palmieri T, Lavrentieva A, Greenhalgh D 2009 An assessment of acute kidney injury with modified RIFLE criteria in pediatric patients with severe burns. Intensive Care Med 35:2125-2129

14. Ronco C, Levin A, Warnock DG, Mehta R, Kellum JA, Shah S, Molitoris BA 2007 Improving outcomes from acute kidney injury (AKI): report on an initiative. Int J Artif Organs 30:373-376 
15. Mathur NB, Agarwal HS, Maria A 2006 Acute renal failure in neonatal sepsis. Indian J Pediatr 73:499-502

16. Abosaif NY, Tolba YA, Heap M, Russell J, El Nahas AM 2005 The outcome of acute renal failure in the intensive care unit according to RIFLE: model application, sensitivity, and predictability. Am J Kidney Dis 46:1038-1048

17. Chertow GM, Soroko SH, Paganini EP, Cho KC, Himmelfarb J, Ikizler TA, Mehta RL 2006 Mortality after acute renal failure: models for prognostic stratification and risk adjustment. Kidney Int 70:1120-1126

18. Ricci Z, Cruz D, Ronco C 2008 The RIFLE criteria and mortality in acute kidney injury: a systematic review. Kidney Int 73:538-546

19. Askenazi DJ, Ambalavanan N, Goldstein SL 2009 Acute kidney injury in critically ill newborns: what do we know? What do we need to learn? Pediatr Nephrol 24:265-274
20. Hasper D, von Haehling S, Storm C, Jorres A, Schefold JC 2009 Changes in serum creatinine in the first 24 hours after cardiac arrest indicate prognosis: an observational cohort study. Crit Care 13:R168

21. Parikh CR, Devarajan P 2008 New biomarkers of acute kidney injury. Crit Care Med 36:S159-S165

22. Bennett M, Dent CL, Ma Q, Dastrala S, Grenier F, Workman R, Syed H, Ali S, Barasch J, Devarajan P 2008 Urine NGAL predicts severity of acute kidney injury after cardiac surgery: a prospective study. Clin J Am Soc Nephrol 3:665-673

23. Dent CL, Ma Q, Dastrala S, Bennett M, Mitsnefes MM, Barasch J, Devarajan P 2007 Plasma neutrophil gelatinase-associated lipocalin predicts acute kidney injury, morbidity and mortality after pediatric cardiac surgery: a prospective uncontrolled cohort study. Crit Care 11:R127

\section{Erratum}

Effect of Obesity on Plasma Clusterin: A Proposed Modulator of Leptin Action: Erratum

In the article that appeared on page 237 of the March 2011 issue, the title of the article was incorrectly presented. The title should have appeared as Effect of Obesity on Plasma Clusterin, a Proposed Modulator of Leptin Action

\section{Reference}

Arnold T, Brandlhofer S, Vrtikapa K, Stangl H, Hermann M, Zwiauer K, Mangge H, Karwautz A, Huemer J, Koller D, Schneider WJ, Strobl W. Effect of Obesity on Plasma Clusterin: A Proposed Modulator of Leptin Action. Pediatr Res 2011;43(3):237-242. 